consistent evaluation will improve the ability to estimate the burden of SLE and enhance efforts to improve HRQOL in SLE.

Disclosure of Interest: E. Hammond Employee of: AstraZeneca, D. Lin: None declared, I. Murimi: None declared, H. Nab Employee of: AstraZeneca, H. Kan Shareholder of: GlaxoSmithKline, O. Onasanya: None declared, J. Tierce: None declared, X. Wang Employee of: AstraZeneca, B. Desta Employee of: AstraZeneca, G. C. Alexander Consultant for: Chair of the FDA's Peripheral and Central Nervous System Advisory Committee, serves as a paid consultant to IQVIA, serves on the Advisory Board of MesaRx Innovations, and serves as a paid member of OptumRx's National P and T Committee. This arrangement has been reviewed and approved by Johns Hopkins University in accordance with its conflict of interest policies.

DOI: 10.1136/annrheumdis-2018-eular.5473

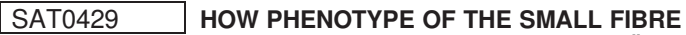 NEUROPATHY (SFN) IN PRIMARY SJÖGREN SYNDROME (PSS) DIFFERS FROM OTHERS CAUSES OF SMALL FIBRE NEUROPATHY?}

E. Descamps $^{1}$, J. Henry ${ }^{1}$, C. Labeyrie ${ }^{2}$, D. Adams ${ }^{2}$, D. Aiello ${ }^{2}$, X. Mariette $^{1}$, R. Seror ${ }^{1} .{ }^{1}$ Rheumatology; ${ }^{2}$ Neurology, Hopitaux Universitaires Paris Sud, Kremlin Bicêtre, France

Background: Small fibre neuropathy (SFN) is a peripheral neuropathy characterised by neuropathic pain associated with normal routine nerve conduction study but rarefaction of intraepidermal nerve fibres (IEFN). Primary Sjögren Syndrome (pSS) is one of the many etiology of SFN.

Objectives: To compare phenotype of SFN in pSS, transthyretin (TTR) familial amyloidosis and idiopathic SFN. To describe evolution of SFN in pSS.

Methods: All patients referred since October 2012 with a biopsy-proven SFN associated with either pSS (ACR/EULAR 2016 criteria), TTR-amyloidosis or idiopathic were included in this monocentric retrospective study. Diagnosis of SFN was confirmed by normal nerve conduction study and abnormal lower limb skin biopsies. All patients undergo standardised diagnosis procedures during an outpatient day-clinic, pSS patients were further followed and undergo a second evaluation. Characteristics of SFN were compared between 3 groups: pSS, TTRamyloidosis and idiopathic, and outcome of pSS associated SFN was analysed. Results: We included 15 patients with pSS (13 (86.7\%) women, median age: 56 years [IQR:46.5-63.5], 7 (46.7\%) anti-SSA positive, 12 (80\%) focus score $\geq 1)$, 17 with TTR-amyloidosis $\left(7(41.2 \%)\right.$ women, median age: 47 years $^{35-}$ $\left.{ }^{56}\right)$ and 11 with idiopathic SFN (7 (63.6\%) women, median age: 47 years [3656.5]). Patients with pSS had a median ESSDAI of $5 .{ }^{5-8}$ One had monoclonal gammopathy, $5 / 13(38.5 \%)$ rheumatoid factor, $2 / 13$ (15.4\%) hypergammaglobulinemia and none had cryoglobulin. Time from first neurologic symptoms to diagnosis of SFN was significantly higher for pSS (29 months [8.5-65]) and idiopathic group (35 months [11.5-65]) than for TTR group (6 months [0-15]). Clinical presentation was length dependant in only $2(13.3 \%)$ patients with pSS compared to $10(58.8 \%)$ in TTR amyloidosis $(p=0.01)$ and $2(18.2 \%)$ in idiopathic group $(p=1)$. A "patchy" presentation (defined by asymmetrical and/or proximal symptoms involving limb, trunk and/or face), was significantly more frequent in pSS than in TTR amyloidosis $(7(43.7 \%)$ vs. $1(5.9 \%) ; p=0.01)$. This more frequent non-length dependant course was confirmed on skin biopsies with an IEFN at proximal site <IEFN at distal site in $7 / 14(50 \%)$ pSS patients compared to $2 / 15(13.3 \%)$ in $\operatorname{TTR}(p=0.05)$ and $1(9.1 \%)$ in idiopathic $(p=0.04)$ groups. Lauria score was significantly higher in pSS than in TTR, $\left(5[4-7.5]\right.$ vs. $\left.2,{ }^{2-5} p=0.007\right)$, mainly due to items of sicca symptoms $(n=14 / 15)$ and peripheral limb pain $(n=13 / 15)$. Ten patients with pSS have been reassessed with a median follow up of 31 months [16.553.5]. At reassessment, the Lauria score did not significantly differ $\left(6^{5-7}\right)$ from initial score, patchy presentation was still predominant $5(50 \%)$. Patients did not evolve through large fibre neuropathy, except one patient who had received a neurotoxic chemotherapy by platin for ovarian cancer, between the 2 evaluations. Conclusions: pSS patients with SFN had a low frequency of serum B cell activation biomarkers. Compared to other causes of SFN, in pSS SNF was characterised by a more frequent non-length dependant and patchy presentation and a higher Lauria score. After a median follow-up of 31 months, SFN in pSS were stable in the time and did not evolve through large fibre neuropathy.

Disclosure of Interest: None declared

DOI: 10.1136/annrheumdis-2018-eular.3298

\section{SAT0430 \\ ASSESSMENT OF ACR AND SLICC CLASSIFICATION CRITERIA IN THE ASIA PACIFIC LUPUS COLLABORATION COHORT}

R. Kandane-Rathnayake ${ }^{1}$, V. Golder ${ }^{1}$, W. Louthrenoo ${ }^{2}$, S.-F. Luo ${ }^{3}$, Y.-J. Wu ${ }^{4}$, A. Lateef ${ }^{5}$, S. Sockalingam ${ }^{6}$, S. Navarra ${ }^{7}$, L. Zamora ${ }^{7}$, L. Hamijoyo ${ }^{8}$,

Y. Katsumata ${ }^{9}$, M. Harigai ${ }^{9}$, M. Chan ${ }^{10}$, S. O'Neill ${ }^{11}$, F. Goldblatt ${ }^{12}$, C.S. Lau ${ }^{13}$, A. Hoi ${ }^{1}$, M. Nikpour ${ }^{14}$, E. Morand ${ }^{1}{ }^{1}$ School of Clinical Sciences at Monash Health, Monash University, Clayton, Australia; ${ }^{2}$ Chiang Mai University Hospital, Chiang Mai, Thailand; ${ }^{3}$ Chang Gung Memorial Hospital, Taipei; ${ }^{4}$ Chang Gung Memorial Hospital, Keelung, Taiwan, Province of China; ${ }^{5}$ National University Hospital, Singapore, Singapore; ${ }^{6}$ University of Malaya, Kuala Lumpur, Malaysia; ${ }^{7}$ University of Santo Tomas Hospital, Manila, Philippines; ${ }^{8}$ University of Padjadjaran, Bandung, Indonesia; ${ }^{9}$ Tokyo Women's Medical University, Tokyo, Japan; ${ }^{10}$ Tan Tock Seng Hospital, Tan Tock Seng, Singapore; ${ }^{11}$ Liverpool Hospital, Liverpool; ${ }^{12}$ Royal Adelaide Hospital and Flinders Medical Centre, Adelaide, Australia; ${ }^{13}$ University of Hong Kong, Pok fu lam, Hong Kong; ${ }^{14}$ St. Vincent's Hospital, Melbourne, Australia

Background: Patients with systemic lupus erythematosus (SLE) are commonly assessed using the classification criteria developed by the American College of Rheumatology (ACR), or more recently by the Systemic Lupus International Collaborating Clinics (SLICC). Although SLE is highly prevalent and severe in Asians, no comparison of patients meeting these criteria in predominantly Asian SLE patients has been performed.

Objectives: To compare patients meeting the ACR and SLICC classification criteria in the Asia Pacific Lupus Collaboration (APLC) cohort.

Methods: All patients fulfilled either the ${ }^{A C R}$ (1997) criteria ( $\geq 4$ of 11 items) or SLICC criteria ( $>4$ of 17 items, including $>1$ clinical and $>1$ immunologic criteria, or biopsy-proven lupus nephritis (LN) $+\geq 1$ immunological criterion), evaluated at enrolment. Demographic and clinical data were compared using Kruskal Wallis (for medians) chi-squared (proportions) tests.

Results: 1735 patients were studied with a median ([IQR] (range)) follow up of $795[532,1087](0,1443)$ days. $1716(98.9 \%)$ and $1668(96.1 \%)$ patients met SLICC and ACR criteria respectively. 1649 (95\%) patients met both criteria, 67 (3.9\%) patients met SLICC criteria only and $19(1.1 \%)$ patients met ACR criteria only. Patients in ACR-only and SLICC-only groups were significantly older than those who met both criteria (ACR-SLICC group); ACR-only patients had longer observation period (table 1). 15/67 SLICC-only patients had non-scarring alopecia, which is not an ACR item, and 14 had $L N$ with $\geq 1$ immunologic criterion. Discrepancies between the 19 ACR-only patients and the ACR-SLICC group were predominantly observed in the immunological criteria. Both ACR-only and SLICConly patients had lower SLEDAI-2k score at recruitment when compared to ACRSLICC group, and a fewer SLICC-only patients were in flare (table 1). During the observation period, SLICC-only patients had the lowest time-adjusted mean (TAM) SLEDAI-2k and prednisolone dose; lowest proportions of flares and damage accrual, and highest proportion of patients achieving Lupus Low Disease Activity State (LLDAS) at least once. In contrast, ACR-only patients had the highest proportion of patients experiencing flares and least proportion of achieving LLDAS (table 1).

\begin{tabular}{|l|ccc|c|}
\hline & $\begin{array}{c}\text { ACR \& SLICC } \\
\mathrm{n}=1649\end{array}$ & $\begin{array}{c}\text { ACR only } \\
\mathrm{n}=19\end{array}$ & $\begin{array}{c}\text { SLICC only } \\
\mathrm{n}=67\end{array}$ & $\mathrm{p}$-value \\
\hline Demographics & median (IQR) & median (IQR) & median (IQR) & \\
Age at enrolment (years) & $40(31,50)$ & $50(37,59)$ & $46(34,55)$ & 0.003 \\
Study observation period (years) & $2.2(1.5,3.0)$ & $3.4(2.4,3.5)$ & $2.1(1.7,2.8)$ & 0.005 \\
Females, $\mathrm{n}(\%)$ & $1533(93.0 \%)$ & $18(94.7 \%)$ & $64(95.5 \%)$ & 0.7 \\
Clinical indications at recruitment & & & & \\
PGA & $0.5(0.2,1.0)$ & $0.5(0.2,0.8)$ & $1.0(0.3,1.2)$ & 0.09 \\
SLEDAl-2k score & $4.0(2.0,6.0)$ & $2.0(0.0,4.0)$ & $2.0(1.0,4.0)$ & 0.03 \\
Mild/mod./severe flare, $\mathrm{n}(\%)$ & $272(16.5 \%)$ & $4(21.1 \%)$ & $3(4.5 \%)$ & 0.03 \\
Organ damage, $\mathrm{n}(\%)$ & $679(41.2 \%)$ & $10(52.6 \%)$ & $25(37.3 \%)$ & 0.5 \\
In LLDAS, $\mathrm{n}(\%)$ & $769(46.7 \%)$ & $7(36.8 \%)$ & $39(58.2 \%)$ & 0.12 \\
Longitudinal clinical indications & & & & \\
Time adjusted mean PGA & $0.4(0.2,0.8)$ & $0.4(0.2,0.6)$ & $0.5(0.2,0.9)$ & 0.7 \\
Time adjusted mean SLEDAI & $3.4(1.5,5.4)$ & $2.6(1.0,5.3)$ & $2.0(1.3,4.0)$ & 0.009 \\
Time adjusted mean prednisolone (mg) & $5.0(2.1,8.9)$ & $4.1(2.5,8.9)$ & $3.0(0.0,6.6)$ & 0.004 \\
Mild/mod./severe flare ever, $\mathrm{n}(\%)$ & $954(57.9 \%)$ & $12(63.2 \%)$ & $21(31.3 \%)$ & $<0.001$ \\
Damage accrual, $\mathrm{n}$ (\%) & $241(14.6 \%)$ & $3(15.8 \%)$ & $6(9.0 \%)$ & 0.4 \\
LLDAS ever, $\mathrm{n}(\%)$ & $1282(77.7 \%)$ & $12(63.2 \%)$ & $58(86.6 \%)$ & 0.07 \\
\hline
\end{tabular}

Conclusions: We observed a high overlap between the two classification criteria but the use of both criteria captured a larger cohort overall. In this cohort, patients meeting SLICC but not ACR criteria had less active disease.

Disclosure of Interest: None declared

DOI: 10.1136/annrheumdis-2018-eular.4308 